\title{
A new class of partially degenerate Hermite-Genocchi polynomials
}

\author{
Waseem A. Khan ${ }^{a}$, Serkan Aracib,*, Mehmet Acikgoz ${ }^{\mathrm{c}}$, Hiba Haroon ${ }^{\mathrm{a}}$ \\ ${ }^{a}$ Department of Mathematics, Faculty of Science, Integral University, Lucknow-226026, India. \\ ${ }^{b}$ Department of Economics, Faculty of Economics, Administrative and Social Science, Hasan Kalyoncu University, TR-27410 Gaziantep, \\ Turkey. \\ ${ }^{c}$ Department of Mathematics, Faculty of Arts and Science, University of Gaziantep, TR-27310 Gaziantep, Turkey.
}

Communicated by S.-H. Rim

\begin{abstract}
In this paper, firstly we introduce not only partially degenerate Hermite-Genocchi polynomials, but also a new generalization of degenerate Hermite-Genocchi polynomials. Secondly, we investigate some behaviors of these polynomials. Furthermore, we establish some implicit summation formulae and symmetry identities by making use of the generating function of partially degenerate Hermite-Genocchi polynomials. Finally, some results obtained here extend well-known summations and identities which we stated in the paper. (C)2017 All rights reserved.
\end{abstract}

Keywords: Hermite polynomials, partially degenerate Genocchi polynomials, partially degenerate Hermite-Genocchi polynomials, summation formula, symmetric identities.

2010 MSC: 11B68, 05A10, 05A15, 33C45.

\section{Introduction}

Throughout the paper, we make use of the following notations:

$$
\mathbb{N}:=\{1,2,3, \cdots\} \text { and } \mathbb{N}_{0}=\mathbb{N} \cup\{0\} .
$$

Let $H_{n}(x, y)$ be denoted by the 2-variable Kampé de Fériet generalization of the Hermite polynomials $[2,3]$ defined as

$$
H_{n}(x, y)=n ! \sum_{r=0}^{\left[\frac{n}{2}\right]} \frac{y^{r} x^{n-2 r}}{r !(n-2 r) !} .
$$

These polynomials are usually defined by the following generating function

$$
e^{x t+y t^{2}}=\sum_{n=0}^{\infty} H_{n}(x, y) \frac{t^{n}}{n !}
$$

and reduce to the ordinary Hermite polynomials $H_{n}(x)$ (see [1]) when $y=-1$ and $x$ is replaced by $2 x$.

\footnotetext{
${ }^{*}$ Corresponding author

Email addresses: waseem08_khan@rediffmail.com (Waseem A. Khan), mtsrkn@hotmail.com (Serkan Araci), acikgoz@gantep.edu.tr (Mehmet Acikgoz), hibaharoon786@gmail.com (Hiba Haroon)

doi:10.22436/jnsa.010.09.43
} 
The classical Bernoulli polynomials $B_{n}(x)$, the classical Euler polynomials $E_{n}(x)$, and the classical Genocchi polynomials $G_{n}(x)$ each of degree $n$ are defined respectively by the following generating functions (see [3-16]):

$$
\begin{aligned}
& \frac{t}{e^{t}-1} e^{x t}=\sum_{n=0}^{\infty} B_{n}(x) \frac{t^{n}}{n !}, \quad(|t|<2 \pi) \\
& \frac{2}{e^{t}+1} e^{x t}=\sum_{n=0}^{\infty} E_{n}(x) \frac{t^{n}}{n !} \quad(|t|<\pi),
\end{aligned}
$$

and

$$
\frac{2 t}{e^{t}+1} e^{x t}=\sum_{n=0}^{\infty} G_{n}(x) \frac{t^{n}}{n !} \quad(|t|<\pi) .
$$

Note that

$$
B_{n}(0)=B_{n}, E_{n}(0)=E_{n} \text {, and } G_{n}(0)=G_{n} \quad(n \in \mathbb{N}) .
$$

The Daehee polynomials are defined by Kim and Kim [5], as follows

$$
\frac{\log (1+t)}{t}(1+t)^{x}=\sum_{n=0}^{\infty} D_{n}(x) \frac{t^{n}}{n !} .
$$

In the case when $x=0$ in (1.1), $D_{n}(0):=D_{n}$ are called the Daehee numbers.

Jang et al. [4] considered the partially degenerate Genocchi polynomials which are given by means of the generating function

$$
\frac{2 \log (1+\lambda t)^{\frac{1}{\lambda}}}{e^{t}+1} e^{x t}=\sum_{n=0}^{\infty} G_{n, \lambda}(x) \frac{t^{n}}{n !} .
$$

In the case when $x=0, G_{n, \lambda}(0):=G_{n, \lambda}$ are called the partially degenerate Genocchi numbers.

Pathan and Khan [13] introduced the generalized Hermite-Bernoulli polynomials for two variables ${ }_{H} B_{n}^{(\alpha)}(x, y)$ given by

$$
\left(\frac{t}{e^{t}-1}\right)^{\alpha} e^{x t+y t^{2}}=\sum_{n=0}^{\infty} H_{n}^{(\alpha)}(x, y) \frac{t^{n}}{n !} .
$$

Taking $\alpha=1$ in (1.3), it reduces to known result of Dattoli et al. [3, p. 386 (1.6)], as follows

$$
\left(\frac{t}{e^{t}-1}\right) e^{x t+y t^{2}}=\sum_{n=0}^{\infty}{ }_{H} B_{n}(x, y) \frac{t^{n}}{n !},
$$

where, for the case $x=y=0$ in (1.4), we have $B_{n}={ }_{H} B_{n}(0,0)$ are called the Bernoulli numbers.

For each $k \in \mathbb{N}_{0}, T_{k}(n)[10]$ defined by

$$
T_{k}(n)=\sum_{j=0}^{n}(-1)^{j} j^{k}
$$

is called the alternating sum. The exponential generating function for $T_{k}(n)$ is

$$
\sum_{k=0}^{\infty} T_{k}(n) \frac{t^{k}}{k !}=\frac{1-\left(-e^{t}\right)^{(n+1)}}{e^{t}+1} .
$$

Recently the special polynomials and degenerate-type special numbers and polynomials have been introduced and studied with applications extensively by many authors (see [1-16] for a systematic work). Inspired and motivated by their works, in this paper, we introduce not only partially degenerate HermiteGenocchi polynomials but also a new generalization of partially degenerate Hermite-Genocchi polynomials and then give some of their applications. We also derive some implicit summation formula and 
general symmetry identities. For obtaining implicit summation formula and general symmetry identities, we use the proof techniques of Dattoli et al. [3], and Pathan and Khan [13].

\section{Partially degenerate Hermite-Genocchi polynomials}

In this section, we assume that $\lambda, t \in \mathbb{C}$ with $|\lambda t| \leqslant 1$ and $\lambda t \neq-1$. Then we consider partially degenerate Hermite-Genocchi polynomials as follows.

$$
\frac{2 \log (1+\lambda t)^{\frac{1}{\lambda}}}{e^{t}+1} e^{x t+y t^{2}}=\sum_{n=0}^{\infty}{ }_{H} G_{n, \lambda}(x, y) \frac{t^{n}}{n !}
$$

so that

$$
{ }_{H} G_{n, \lambda}(x, y)=\sum_{m=0}^{n}\left(\begin{array}{l}
n \\
m
\end{array}\right) G_{m, \lambda} H_{n-m}(x, y) .
$$

In the case $x=y=0$ in (2.1), we have ${ }_{H} G_{n, \lambda}(0,0):=G_{n, \lambda}$ are called the partially degenerate Genocchi numbers introduced by Jang et al. [4].

Theorem 2.1. For $\mathrm{n} \in \mathbb{N}_{0}$, we have

$$
{ }_{H} G_{n, \lambda}(x, y)=\sum_{m=0}^{n}(n)_{m} m !(-\lambda)^{m}{ }_{H} G_{n-m}(x, y) .
$$

Proof. It follows from (2.1) that

$$
\begin{aligned}
\sum_{n=0}^{\infty}{ }_{H} G_{n, \lambda}(x, y) \frac{t^{n}}{n !} & =\frac{2 \log (1+\lambda t)^{\frac{1}{\lambda}}}{e^{t}+1} e^{x t+y t^{2}} \\
& =\left(\sum_{m=0}^{\infty} \frac{(-1)^{m}}{m+1}(\lambda t)^{m}\right)\left(\sum_{n=0}^{\infty} H_{n}(x, y) \frac{t^{n}}{n !}\right) \\
& =\sum_{n=0}^{\infty}\left(\sum_{m=0}^{n}\left(\begin{array}{c}
n \\
m
\end{array}\right) \frac{(-\lambda)^{m}}{m+1} m !_{H} G_{n-m}(x, y)\right) \frac{t^{n}}{n !}
\end{aligned}
$$

Matching the coefficients $\frac{t^{n}}{n !}$ gives the desired result.

Remark 2.2. In the case when $y=0$ in Theorem 2.1, our result reduces to the result of Jang et al. [4, p.4 (13)].

Theorem 2.3. For $\mathrm{n} \in \mathbb{N}_{0}$, we have

$$
{ }_{H} G_{n+1, \lambda}(x, y)=\sum_{m=0}^{n+1}\left(\begin{array}{c}
n+1 \\
m
\end{array}\right) \lambda^{m}{ }_{H} G_{n-m+1}(x, y) D_{m} .
$$

Proof. We first consider

$$
\begin{aligned}
I_{1}=\frac{1}{t} \frac{2 \log (1+\lambda t)^{\frac{1}{\lambda}}}{e^{t}+1} e^{x t+y t^{2}} & =\left(\sum_{m=0}^{\infty} D_{m} \frac{(\lambda t)^{m}}{m !}\right)\left(\sum_{n=0}^{\infty} H_{n}(x, y) \frac{t^{n}}{n !}\right) \\
& =\sum_{n=1}^{\infty}\left(\sum_{m=0}^{n}\left(\begin{array}{c}
n \\
m
\end{array}\right) \lambda^{m} D_{m H} G_{n-m}(x, y)\right) \frac{t^{n}}{n !} \\
& =t \sum_{n=0}^{\infty}\left(\sum_{m=0}^{n+1}\left(\begin{array}{c}
n+1 \\
m
\end{array}\right) \lambda^{m} D_{m} \frac{H G_{n-m+1}(x, y)}{n+1}\right) \frac{t^{n}}{n !}
\end{aligned}
$$


Secondly

$$
I_{2}=\frac{1}{t} \frac{2 \log (1+\lambda t)^{\frac{1}{\lambda}}}{e^{t}+1} e^{x t+y t^{2}}=\frac{1}{t} \sum_{n=0}^{\infty} H_{n, \lambda}(x, y) \frac{t^{n}}{n !}=\sum_{n=0}^{\infty} \frac{{ }_{H} G_{n+1, \lambda}(x, y)}{n+1} \frac{t^{n}}{n !} .
$$

Since $I_{1}=I_{2}$, we thus conclude the proof.

Remark 2.4. Taking $y=0$ in Theorem 2.3 gives the result of Jang et al. [4, p.5 (19)].

Theorem 2.5. For $\mathrm{n} \in \mathbb{N}_{0}$, we have

$$
{ }_{H} G_{n, \lambda}(x, y)=n \sum_{m=0}^{n-1}\left(\begin{array}{c}
n-1 \\
m
\end{array}\right) \lambda^{m}{ }_{H} E_{n-m-1}(x, y) D_{m} .
$$

Proof. From (2.1), we can write

$$
\begin{aligned}
\sum_{n=0}^{\infty}{ }_{H} G_{n, \lambda}(x, y) \frac{t^{n}}{n !} & =t \frac{\log (1+\lambda t)}{\lambda t} \frac{2}{e^{t}+1} e^{x t+y t^{2}} \\
& =t\left(\sum_{m=0}^{\infty} D_{m} \frac{(\lambda t)^{m}}{m !}\right)\left(\sum_{n=0}^{\infty} H E_{n}(x, y) \frac{t^{n}}{n !}\right) \\
& =\sum_{n=0}^{\infty}\left(\sum_{m=0}^{n}\left(\begin{array}{c}
n \\
m
\end{array}\right) \lambda^{m} D_{m H} E_{n-m}(x, y)\right) \frac{t^{n+1}}{n !} .
\end{aligned}
$$

Thus, comparing the coefficients $t^{n}$ in the both sides on the above, we end the proof.

Remark 2.6. Putting $y=0$ in Theorem 2.5 yields to known result of Jang et al. [4, p.5 (21)].

Theorem 2.7. For $\mathrm{n} \in \mathbb{N}_{0}$, we obtain

$$
{ }_{H} G_{n, \lambda}(x+1, y)=\sum_{m=0}^{n}\left(\begin{array}{l}
n \\
m
\end{array}\right)\left({ }_{H} G_{n-m, \lambda}(x, y)\right) .
$$

Proof. By making use of Eq. (2.1), we see that

$$
\begin{aligned}
\sum_{n=0}^{\infty}\left({ }_{H} G_{n, \lambda}(x+1, y)-{ }_{H} G_{n, \lambda}(x, y)\right) \frac{t^{n}}{n !} & =\frac{2 \log (1+\lambda t)^{\frac{1}{\lambda}}}{e^{t}+1} e^{(x+1) t+y t^{2}}-\frac{2 \log (1+\lambda t)^{\frac{1}{\lambda}}}{e^{t}+1} e^{x t+y t^{2}} \\
& =\sum_{n=0}^{\infty} H_{n, \lambda}(x, y) \frac{t^{n}}{n !} \sum_{m=0}^{\infty} \frac{t^{m}}{m !}-\sum_{n=0}^{\infty}{ }_{H} G_{n, \lambda}(x, y) \frac{t^{n}}{n !} \\
& =\sum_{n=0}^{\infty}\left(\sum_{m=0}^{n}\left(\begin{array}{c}
n \\
m
\end{array}\right){ }_{H} G_{n-m, \lambda}(x, y)-{ }_{H} G_{n, \lambda}(x, y)\right) \frac{t^{n}}{n !} .
\end{aligned}
$$

Comparing the coefficients $\frac{t^{n}}{n !}$ in both sides of the above equation, we get the result (2.2).

Corollary 2.8. In the case when $y=0$ in Theorem 2.7, one can see

$$
\mathrm{G}_{\mathrm{n}, \lambda}(x+1)=\sum_{m=0}^{n}\left(\begin{array}{l}
n \\
m
\end{array}\right) G_{n-m, \lambda}(x) .
$$

Theorem 2.9. For $\mathrm{n} \in \mathbb{N}_{0}$, we have

$$
{ }_{H} G_{n, \lambda}(x, y)=\sum_{m=0}^{n} \sum_{k=0}^{m}\left(\begin{array}{l}
n \\
m
\end{array}\right)\left(\begin{array}{c}
m \\
k
\end{array}\right) G_{n-m} D_{m-k} \lambda^{m-k} H_{k}(x, y) .
$$


Proof. Since

$$
\begin{aligned}
\sum_{n=0}^{\infty} H_{n, \lambda}(x, y) \frac{t^{n}}{n !} & =\frac{2 \log (1+\lambda t)^{\frac{1}{\lambda}}}{e^{t}+1} e^{x t+y t^{2}} \\
& =\left(\frac{2 t}{e^{t}+1}\right)\left(\frac{\log (1+\lambda t)}{\lambda t}\right) e^{x t+y t^{2}} \\
& =\left(\sum_{n=0}^{\infty} G_{n} \frac{t^{n}}{n !}\right)\left(\sum_{m=0}^{\infty} D_{m} \frac{(\lambda t)^{m}}{m !}\right)\left(\sum_{k=0}^{\infty} H_{k}(x, y) \frac{t^{k}}{k !}\right),
\end{aligned}
$$

we have

$$
=\sum_{n=0}^{\infty}\left(\sum_{m=0}^{n} \sum_{k=0}^{m}\left(\begin{array}{c}
n \\
m
\end{array}\right)\left(\begin{array}{c}
m \\
k
\end{array}\right) G_{n-m} D_{m-k} \lambda^{m-k} H_{k}(x, y)\right) \frac{t^{n}}{n !} .
$$

We thus complete the proof.

We now give a multiplication formula for partially degenerate Hermite-Genocchi polynomials.

Theorem 2.10. For $\mathrm{n} \in \mathbb{N}_{0}$, we have

$$
{ }_{H} G_{n, \lambda}(x, y)=d^{n-1} \sum_{a=0}^{d-1} H_{n, \frac{\lambda}{d}}\left(\frac{a+x}{d}, y\right) .
$$

Proof. From (2.1), we have

$$
\begin{aligned}
\sum_{n=0}^{\infty} H G_{n, \lambda}(x, y) \frac{t^{n}}{n !}=\frac{2 \log (1+\lambda t)^{\frac{1}{\lambda}}}{e^{t}+1} e^{x t+y t^{2}} & =\frac{2 \log (1+\lambda t)^{\frac{1}{\lambda}}}{e^{d t}+1} e^{y t^{2}} \sum_{a=0}^{d-1} e^{(a+x) t} \\
& =\sum_{n=0}^{\infty}\left(d^{n-1} \sum_{a=0}^{d-1} H G_{n, \frac{\lambda}{d}}\left(\frac{a+x}{d}, y\right)\right) \frac{t^{n}}{n !} .
\end{aligned}
$$

Equating the coefficients $\frac{\mathrm{t}^{\mathrm{n}}}{\mathrm{n} !}$ of the both sides of above equation, we arrive at the desired result.

Corollary 2.11. In the case $y=0$, one can get

$$
G_{n, \lambda}(x)=d^{n-1} \sum_{a=0}^{d-1} G_{n, \frac{\lambda}{d}}\left(\frac{x+a}{d}\right) .
$$

\section{Generalized partially degenerate Hermite-Genocchi polynomials}

Let $d \in \mathbb{N}$ with $d \equiv 1(\bmod 2)$ and $\chi$ be a Dirichlet character with conductor $d$. We consider the generalized partially degenerate Hermite-Genocchi polynomials attached to $\chi$ by means of the following generating function:

$$
\sum_{n=0}^{\infty} H_{n, \chi, \lambda}(x, y) \frac{t^{n}}{n !}=\frac{2 \log (1+\lambda t)^{\frac{1}{\lambda}}}{e^{d t}+1} \sum_{a=0}^{d-1}(-1)^{a} \chi(a) e^{(a+x) t+y t^{2}} .
$$

When $x=y=0$ in (3.1), we have $G_{n, \chi, \lambda}={ }_{H} G_{n, x, \lambda}(0,0)$ that stands for the generalized partially degenerate Genocchi numbers attached to $\chi$. Also we observe that

$$
\lim _{\substack{x \rightarrow 0 \\ y=0}}{ } G_{n, \chi, \lambda}(x, y)=G_{n, \chi}(x)
$$

is generalized Genocchi polynomial (see [15]). 
Theorem 3.1. For $\mathrm{n} \in \mathbb{N}_{0}$, we have

$$
{ }_{H} G_{n, \chi, \lambda}(x, y)=\sum_{m=0}^{n}\left(\begin{array}{l}
n \\
m
\end{array}\right) \lambda^{m} D_{m H} G_{n-m, x}(x, y) .
$$

Proof. It follows from (3.1) that

$$
\begin{aligned}
\sum_{n=0}^{\infty}{ }_{H} G_{n, \chi, \lambda}(x, y) \frac{t^{n}}{n !} & =\frac{2 \log (1+\lambda t)^{\frac{1}{\lambda}}}{e^{d t}+1} \sum_{a=0}^{d-1}(-1)^{a} \chi(a) e^{(a+x) t+y t^{2}} \\
& =\left(\frac{\log (1+\lambda t)}{\lambda t}\right)\left(\frac{2 t}{e^{d t}+1} \sum_{a=0}^{d-1}(-1)^{a} \chi(a) e^{(a+x) t+y t^{2}}\right) \\
& =\left(\sum_{m=0}^{\infty} D_{m} \frac{\lambda^{m} t^{m}}{m !}\right)\left(\sum_{n=0}^{\infty} H G_{n, \chi}(x, y) \frac{t^{n}}{n !}\right)
\end{aligned}
$$

Comparing the coefficients of $\frac{t^{n}}{n !}$ on both sides of the above equation, we complete the proof.

Theorem 3.2. The following equality

$$
{ }_{H} G_{n, \chi, \lambda}(x, y)=d^{n-1} \sum_{a=0}^{d-1}(-1)^{a} \chi(a)_{H} G_{n, \frac{\lambda}{d}}\left(\frac{a+x}{d}, y\right)
$$

holds true.

Proof. We consider

$$
\begin{aligned}
\sum_{n=0}^{\infty} H_{n, \chi, \lambda}(x, y) \frac{t^{n}}{n !} & =\frac{2 \log (1+\lambda t)^{\frac{1}{\lambda}}}{e^{d t}+1} \sum_{a=0}^{d-1}(-1)^{a} \chi(a) e^{(a+x) t+y t^{2}} \\
& =\frac{1}{d} \sum_{a=0}^{d-1}(-1)^{a} \chi(a) \frac{2 \log (1+\lambda t)^{\frac{d}{\lambda}}}{e^{d t}+1} e^{\left(\frac{a+x}{d}\right) d t+y t^{2}} \\
& =\sum_{n=0}^{\infty}\left(d^{n-1} \sum_{a=0}^{d-1}(-1)^{a} \chi(a)_{H} G_{n, \frac{\lambda}{d}}\left(\frac{a+x}{d}, y\right)\right) \frac{t^{n}}{n !} .
\end{aligned}
$$

Equating the coefficients $\frac{\mathrm{t}^{\mathrm{n}}}{\mathrm{n} !}$ on both sides of the above equation yields the proof of this theorem.

By using Eq. (3.1), the proofs of the following theorems in this section can be shown easily. So we omit the proofs.

Theorem 3.3. The following

$$
{ }_{H} G_{n, \chi, \lambda}(x, y)=\sum_{m=0}^{\left[\frac{\mathfrak{n}]}{2}\right.} \frac{n !}{(n-2 m) ! m !} G_{n-2 m, x, \lambda}(x) y^{m}
$$

holds true.

Theorem 3.4. The following

$$
\frac{H G_{n, \chi, \lambda}(x, y)}{n !}=\sum_{l=0}^{n} \sum_{m=0}^{\left[\begin{array}{c}
\frac{n}{2} \\
m
\end{array}\right.} \frac{\left(\begin{array}{c}
n-2 m \\
l
\end{array}\right)}{(n-2 m) ! m !} G_{l, x, \lambda} x^{n-2 m-l} y^{m}
$$

holds true. 


\section{Implicit formula involving partially degenerate Hermite-Genocchi polynomials}

In this section we give implicit formula of partially degenerate Hermite-Genocchi polynomials by making use of generating function technique.

Theorem 4.1. The following implicit summation formula for partially degenerate Hermite-Genocchi polynomials ${ }_{H} G_{n, \lambda}(x, y)$ holds true:

$$
{ }_{H} G_{k+l, \lambda}(z, y)=\sum_{n, p=0}^{k, l}\left(\begin{array}{l}
k \\
n
\end{array}\right)\left(\begin{array}{l}
l \\
p
\end{array}\right)(z-x)^{n+p}{ }_{H} G_{k+l-p-n, \lambda}(x, y) .
$$

Proof. We first replace $t$ by $t+u$ and rewrite the generating function (2.1) as

$$
\frac{2 \log (1+\lambda(t+u))^{\frac{1}{\lambda}}}{e^{(t+u)}+1} e^{y(t+u)^{2}}=e^{-x(t+u)} \sum_{k, l=0}^{\infty}{ }_{H} G_{k+l, \lambda}(x, y) \frac{t^{k} u^{l}}{k ! l !} \quad \text { see [13]). }
$$

Replacing $x$ by $z$ in the above equation and equating the resulting equation to the above equation, we have

$$
e^{(z-x)(t+u)} \sum_{k, l=0}^{\infty}{ }_{H} G_{k+l, \lambda}(x, y) \frac{t^{k}}{k !} \frac{u^{l}}{l !}=\sum_{k, l=0}^{\infty}{ }_{H} G_{k+l, \lambda}(z, y) \frac{t^{k}}{k !} \frac{u^{l}}{l !}
$$

and also

$$
\sum_{N=0}^{\infty} \frac{[(z-x)(t+u)]^{N}}{N !} \sum_{k, l=0}^{\infty}{ }_{H} G_{k+l, \lambda}(x, y) \frac{t^{k}}{k !} \frac{u^{l}}{l !}=\sum_{k, l=0}^{\infty}{ }_{H} G_{k+l, \lambda}(z, y) \frac{t^{k}}{k !} \frac{u^{l}}{l !},
$$

where we have used the following formula taken in [16, p.52 (2)]

$$
\sum_{N=0}^{\infty} f(N) \frac{(x+y)^{N}}{N !}=\sum_{n, m=0}^{\infty} f(n+m) \frac{x^{n}}{n !} \frac{y^{m}}{m !}
$$

In the left hand side of Eq. (4.2), it becomes

$$
\sum_{n, p=0}^{\infty} \frac{(z-x)^{n+p} t^{n} u^{p}}{n ! p !} \sum_{k, l=0}^{\infty}{ }_{H} G_{k+l, \lambda}(x, y) \frac{t^{k}}{k !} \frac{u^{l}}{l !}=\sum_{k, l=0}^{\infty}{ }_{H} G_{k+l, \lambda}(z, y) \frac{t^{k}}{k !} \frac{u^{l}}{l !} .
$$

Now replacing $k$ by $k-n, l$ by $l-p$, and using the lemma [16, p.100 (1)] in the left hand side of (4.3), we get

$$
\sum_{n, p=0}^{\infty} \sum_{k, l=0}^{n, p} \frac{(z-x)^{n+p}}{n ! p !} H_{k+l-n-p, \lambda}(x, y) \frac{t^{k}}{(k-n) !} \frac{u^{l}}{(l-p) !}=\sum_{k, l=0}^{\infty}{ }_{H} G_{k+l, \lambda}(z, y) \frac{t^{k}}{k !} \frac{u^{l}}{l !} .
$$

Thus, on equating the coefficients of the like powers of $t^{k}$ and $u^{l}$ in the above equation, we arrive at the desired result.

Corollary 4.2. In the case $\mathrm{l}=0$ in Eq. (4.1), we have

$$
{ }_{H} G_{k, \lambda}(z, y)=\sum_{j=0}^{k}\left(\begin{array}{l}
k \\
j
\end{array}\right)(z-x)^{j}{ }_{H} G_{k-j, \lambda}(x, y) .
$$

Note that for special values of the parameters $y$ and $z$ in Theorem 4.1, one can obtain some identities of usual Genocchi polynomials. Now we also state some theorems including this section which can be easily proved by making use of Eq. (2.1). So we give theorems without proof. 
Theorem 4.3. The following implicit summation formula for partially degenerate Hermite-Genocchi polynomials ${ }_{H} G_{n, \lambda}(x, y)$ holds true:

$$
{ }_{H} G_{n, \lambda}(x+z, y+u)=\sum_{m=0}^{n}\left(\begin{array}{c}
n \\
m
\end{array}\right) H G_{n-m, \lambda}(z, u) H_{m}(x, y) .
$$

Theorem 4.4. The following implicit summation formula for partially degenerate Hermite-Genocchi polynomials ${ }_{H} G_{n, \lambda}(x, y)$ holds true:

$$
{ }_{H} G_{n, \lambda}(y, x)=n ! \sum_{k=0}^{\frac{n}{2}} G_{n-2 k, \lambda}(y) \frac{x^{k}}{(n-2 k) ! k !} .
$$

Theorem 4.5. The following implicit summation formula for partially degenerate Hermite-Genocchi polynomials ${ }_{H} G_{n, \lambda}(x, y)$ holds true:

$$
{ }_{H} G_{n, \lambda}(x, y)=\sum_{m=0}^{n}\left(\begin{array}{l}
n \\
m
\end{array}\right) G_{n-m, \lambda}(x-z) H_{m}(z, y) \text {. }
$$

Theorem 4.6. The following implicit summation formula for partially degenerate Hermite-Genocchi polynomials ${ }_{H} G_{n, \lambda}(x, y)$ holds true:

$$
{ }_{H} G_{n, \lambda}(x+1, y)=\sum_{m=0}^{n}\left(\begin{array}{l}
n \\
m
\end{array}\right) H G_{n-m, \lambda}(x, y) .
$$

Theorem 4.7. The following implicit summation formula for partially degenerate Hermite-Genocchi polynomials ${ }_{H} G_{n, \lambda}(x, y)$ holds true:

$$
{ }_{H} G_{n, \lambda}(x+1, y)+{ }_{H} G_{n, \lambda}(x, y)=2 n \sum_{m=0}^{n-1}\left(\begin{array}{c}
n-1 \\
m
\end{array}\right) \frac{(-\lambda)^{m} m !}{m+1} H_{n-1-m}(x, y) .
$$

\section{Symmetry identities for partially degenerate Hermite-Genocchi polynomials}

In this section, we give general symmetry identities for the partially degenerate Hermite-Genocchi polynomials ${ }_{H} G_{n, \lambda}(x, y)$ by making use of the generating functions (1.2) and (2.1).

Theorem 5.1. For each pair of integers $\mathrm{a}$ and $\mathrm{b}$ with $\mathrm{n} \geqslant 0$, the following symmetry identity holds true:

$$
\begin{aligned}
& \sum_{m=0}^{n}\left(\begin{array}{c}
n \\
m
\end{array}\right) b^{m} a^{n-m}{ }_{H} G_{n-m, \lambda}\left(b x, b^{2} y\right)_{H} G_{m, \lambda}\left(a x, a^{2} y\right) \\
& \quad=\sum_{m=0}^{n}\left(\begin{array}{l}
n \\
m
\end{array}\right) a^{m} b^{n-m_{H}} G_{n-m, \lambda}\left(a x, a^{2} y\right)_{H} G_{m, \lambda}\left(b x, b^{2} y\right) .
\end{aligned}
$$

Proof. We first consider

$$
g(t)=\frac{\left(2 \log (1+\lambda t)^{\frac{a}{\lambda}}\right)\left(2 \log (1+\lambda t)^{\frac{b}{\lambda}}\right)}{\left(e^{a t}+1\right)\left(e^{b t}+1\right)} e^{a b x t+a^{2} b^{2} y t^{2}}
$$

where $g(t)$ is symmetric in $a$ and $b$, and can be expressed into series in two ways. 
On the one hand,

$$
\begin{aligned}
g(t) & =\sum_{n=0}^{\infty} H_{n, \lambda}\left(b x, b^{2} y\right) \frac{(a t)^{n}}{n !} \sum_{m=0}^{\infty}{ }_{H} G_{m, \lambda}\left(a x, a^{2} y\right) \frac{(b t)^{m}}{m !} \\
& =\sum_{n=0}^{\infty}\left(\sum_{m=0}^{n}\left(\begin{array}{l}
n \\
m
\end{array}\right) b^{m} a^{n-m}{ }_{H} G_{n-m, \lambda}\left(b x, b^{2} y\right)_{H} G_{m, \lambda}\left(a x, a^{2} y\right)\right) \frac{t^{n}}{n !},
\end{aligned}
$$

and on the other hand,

$$
\begin{aligned}
g(t) & =\sum_{n=0}^{\infty} H_{n, \lambda}\left(a x, a^{2} y\right) \frac{(b t)^{n}}{n !} \sum_{m=0}^{\infty} H_{m, \lambda}\left(b x, b^{2} y\right) \frac{(a t)^{m}}{m !} \\
& =\sum_{n=0}^{\infty}\left(\sum_{m=0}^{n}\left(\begin{array}{l}
n \\
m
\end{array}\right) a^{m} b^{n-m_{H}} G_{n-m, \lambda}\left(a x, a^{2} y\right)_{H} G_{m, \lambda}\left(b x, b^{2} y\right)\right) \frac{t^{n}}{n !} .
\end{aligned}
$$

By comparing the coefficients $t^{n}$ on the right hand sides of Eqs. (5.1) and (5.2), we get the proof of theorem.

Theorem 5.2. For each pair of integers $\mathrm{a}$ and $\mathrm{b}$ with $\mathrm{n} \geqslant 1$, the following symmetry identity holds true:

$$
\begin{aligned}
& \sum_{k=0}^{n}\left(\begin{array}{l}
n \\
k
\end{array}\right) a^{n-k} b^{k} \sum_{i=0}^{a-1} \sum_{j=0}^{b-1}(-1)^{i+j}{ }_{H} G_{n-k, \lambda}\left(b x+\frac{b}{a} i+j, b^{2} z\right) G_{k, \lambda}(a y) \\
& =\sum_{k=0}^{n}\left(\begin{array}{l}
n \\
k
\end{array}\right) b^{n-k} a^{k} \sum_{i=0}^{b-1} \sum_{j=0}^{a-1}(-1)^{i+j}{ }_{H} G_{n-k, \lambda}\left(a x+\frac{a}{b} i+j, a^{2} z\right) G_{k, \lambda}(b y) .
\end{aligned}
$$

Proof. Let

$$
g(t)=\frac{\left(2 \log (1+\lambda t)^{\frac{a}{\lambda}}\right)\left(2 \log (1+\lambda t)^{\frac{b}{\lambda}}\right)\left(e^{a b t}+1\right)^{2}}{\left(e^{a t}+1\right)^{2}\left(e^{b t}+1\right)^{2}} e^{a b(x+y) t+a^{2} b^{2} z t^{2}} .
$$

We consider $\mathrm{g}(\mathrm{t})$ with two ways. Firstly,

$$
\begin{aligned}
g(t)= & \frac{2 \log (1+\lambda t)^{\frac{a}{\lambda}}}{e^{a t}+1} e^{a b x t+a^{2} b^{2} z t^{2}}\left(\frac{e^{a b t}+1}{e^{b t}+1}\right) \frac{2 \log (1+\lambda t)^{\frac{b}{\lambda}}}{e^{b t}+1} e^{a b y t}\left(\frac{e^{a b t}+1}{e^{a t}+1}\right) \\
= & \frac{2 \log (1+\lambda t)^{\frac{a}{\lambda}}}{e^{a t}+1} e^{a b x t+a^{2} b^{2} z t^{2}}\left(\sum_{i=0}^{a-1}(-1)^{i} e^{b t i}\right) \frac{2 \log (1+\lambda t)^{\frac{b}{\lambda}}}{e^{b t}+1} e^{a b y t}\left(\sum_{j=0}^{b-1}(-1)^{j} e^{a t j}\right) \\
= & \frac{2 \log (1+\lambda t)^{\frac{a}{\lambda}}}{e^{a t}+1} e^{a^{2} b^{2} z t^{2}}\left(\sum_{i=0}^{a-1} \sum_{j=0}^{b-1}(-1)^{i+j} e^{\left(b x+\frac{b}{a} i+j\right) a t}\right)\left(\sum_{k=0}^{\infty} G_{k, \lambda}(a y) \frac{(b t)^{k}}{k !}\right) \\
= & \left(\sum_{n=0}^{\infty} \sum_{i=0}^{a-1} \sum_{j=0}^{b-1}(-1)^{i+j} G_{n, \lambda}\left(b x+\frac{b}{a} i+j, b^{2} z\right) \frac{(a t)^{n}}{n !}\right)\left(\sum_{k=0}^{\infty} G_{k, \lambda}(a y) \frac{(b t)^{k}}{(k) !}\right) \\
& \times \sum_{n=0}^{\infty}\left(\sum_{k=0}^{n}\left(\begin{array}{l}
n \\
k
\end{array}\right) a^{n-k} b^{k} \sum_{i=0}^{a-1} \sum_{j=0}^{b-1}(-1)^{i+j} G_{n-k, \lambda}\left(b x+\frac{b}{a} i+j, b^{2} z\right) G_{k, \lambda}(a y)\right) \frac{t^{n}}{n !} .
\end{aligned}
$$

Secondly,

$$
g(t)=\sum_{n=0}^{\infty}\left(\sum_{k=0}^{n}\left(\begin{array}{l}
n \\
k
\end{array}\right) b^{n-k} a^{k} \sum_{i=0}^{b-1} \sum_{j=0}^{a-1}(-1)^{i+j}{ }_{H} G_{n-k, \lambda}\left(a x+\frac{a}{b} i+j, a^{2} z\right) G_{k, \lambda}(b y)\right) \frac{t^{n}}{n !} .
$$

Since two ways are equal, we arrive at the desired result by comparing the coefficients $\frac{t^{n}}{n !}$. 
We now give the following two theorems without proofs because their proofs techniques are the same with the above theorems of the final section of this paper.

Theorem 5.3. For each pair of integers $\mathrm{a}$ and $\mathrm{b}$ and all integers $\mathrm{n} \geqslant 0$, the following symmetry identity holds true:

$$
\begin{aligned}
& \sum_{k=0}^{n}\left(\begin{array}{l}
n \\
k
\end{array}\right) a^{n-k} b^{k} \sum_{i=0}^{a-1} \sum_{j=0}^{b-1}(-1)^{i+j}{ }_{H} G_{n-k, \lambda}\left(b x+\frac{b}{a} i, b^{2} z\right) G_{k, \lambda}\left(a y+\frac{a}{b} j\right) \\
& =\sum_{k=0}^{n}\left(\begin{array}{l}
n \\
k
\end{array}\right) b^{n-k} a^{k} \sum_{i=0}^{b-1} \sum_{j=0}^{a-1}(-1)^{i+j}{ }_{H} G_{n-k, \lambda}\left(a x+\frac{a}{b} i, a^{2} z\right) G_{k, \lambda}\left(b y+\frac{b}{a} j\right) .
\end{aligned}
$$

Theorem 5.4. For each pair of integers $\mathrm{a}$ and $\mathrm{b}$ and all integers $\mathrm{n} \geqslant 0$, the following symmetry identity holds true:

$$
\begin{aligned}
& \sum_{k=0}^{n}\left(\begin{array}{l}
n \\
k
\end{array}\right) a^{n-k} b^{k}{ }_{H} G_{n-k, \lambda}\left(b x, b^{2} z\right) \sum_{i=0}^{k}\left(\begin{array}{l}
k \\
i
\end{array}\right) T_{i}(a-1) G_{k-i, \lambda}(a y) \\
& =\sum_{k=0}^{n}\left(\begin{array}{l}
n \\
k
\end{array}\right) a^{k} b^{n-k}{ }_{H} G_{n-k, \lambda}\left(a x, a^{2} z\right) \sum_{i=0}^{k}\left(\begin{array}{l}
k \\
i
\end{array}\right) T_{i}(b-1) G_{k-i, \lambda}(b y),
\end{aligned}
$$

where the sum of alternative integer powers $T_{k}(n)$ is already given by Eq. (1.5).

\section{References}

[1] L. C. Andrews, Special functions for engineers and mathematicians, Macmillan Co., New York, (1985). 1, 1

[2] E. T. Bell, Exponential polynomials, Ann. of Math., 35 (1934), 258-277. 1

[3] G. Dattoli, S. Lorenzutta, C. Cesarano, Finite sums and generalized forms of Bernoulli polynomials, Rend. Mat. Appl., 19 (1999), 385-391. 1, 1, 1, 1

[4] L. C. Jang, H. I. Kwon, J. G. Lee, C. S. Ryoo, On the generalized partially degenerate Genocchi polynomials, Global J. Pure Appl. Math., 11 (2015), 4789-4799. 1, 2, 2.2, 2.4, 2.6

[5] D. S. Kim, T. Kim, Daehee numbers and polynomials, Appl. Math. Sci., 7 (2013), 5969-5976. 1

[6] D. S. Kim, T. Kim, Some identities of degenerate special polynomials, Open Math., 13 (2015), 380-389.

[7] D. S. Kim, T. Kim, S.-H. Lee, J.-J. Seo, A note on the lambda-Daehee polynomials, Int. J. Math. Anal., 7 (2013), 3069 3080 .

[8] T. Kim, S. H. Lee, T. Mansour, J. J. Seo, A note on q-Daehee polynomials and numbers, Adv. Stud. Contemp. Math., 24 (2014), 155-160.

[9] T. Kim, J. J. Seo, A note on partially degenerate Bernoulli numbers and polynomials, J. Math. Anal., 6 (2015), 1-6.

[10] D. Lim, Degenerate, partially degenerate and totally degenerate Daehee numbers and polynomials, Adv. Difference Equ., 2015 (2015), 14 pages. 1

[11] D. Lim, Some identities of degenerate Genocchi polynomials, Bull. Korean Math. Soc., 53 (2016), 569-579.

[12] J.-W. Park, J. Kwon, A Note on the Degenerate High Order Daehee Polynomials, Applied Mathematical Sciences, 9 (2015), 4635-4642.

[13] M. A. Pathan, W. A. Khan, Some implicit summation formulas and symmetric identities for the generalized HermiteBernoulli polynomials, Mediterr. J. Math., 12 (2015), 679-695. 1, 1, 4.2

[14] F. Qi, D. V. Dolgy, T. Kim, C. S. Ryoo, On the partially degenerate Bernoulli polynomials of the first kind, Global J. Pure Appl. Math., 11 (2015), 2407-2412.

[15] C. S. Ryoo, T. Kim, J. Choi, B. Lee, On the Generalized q-Genochi Numbers and Polynomials of Higher-Order, Adv. Difference Equ., 2011 (2011), 8 pages. 3

[16] H. J. H. Tuenter, A symmetry power sum of polynomials and Bernoulli numbers, Amer. Math. Monthly, 108 (2001), 258-261. 1, 1, 4, 4 